\title{
Equal-budget choice equivalent solutions in exchange economies
}

Citation for published version (APA):

Bochet, O. L. A. (2006). Equal-budget choice equivalent solutions in exchange economies. METEOR, Maastricht University School of Business and Economics. METEOR Research Memorandum No. 025 https://doi.org/10.26481/umamet.2006025

Document status and date:

Published: 01/01/2006

DOI:

10.26481/umamet.2006025

Document Version:

Publisher's PDF, also known as Version of record

\section{Please check the document version of this publication:}

- A submitted manuscript is the version of the article upon submission and before peer-review. There can be important differences between the submitted version and the official published version of record.

People interested in the research are advised to contact the author for the final version of the publication, or visit the DOI to the publisher's website.

- The final author version and the galley proof are versions of the publication after peer review.

- The final published version features the final layout of the paper including the volume, issue and page numbers.

Link to publication

\footnotetext{
General rights rights.

- You may freely distribute the URL identifying the publication in the public portal. please follow below link for the End User Agreement:

www.umlib.nl/taverne-license

Take down policy

If you believe that this document breaches copyright please contact us at:

repository@maastrichtuniversity.nl

providing details and we will investigate your claim.
}

Copyright and moral rights for the publications made accessible in the public portal are retained by the authors and/or other copyright owners and it is a condition of accessing publications that users recognise and abide by the legal requirements associated with these

- Users may download and print one copy of any publication from the public portal for the purpose of private study or research.

- You may not further distribute the material or use it for any profit-making activity or commercial gain

If the publication is distributed under the terms of Article $25 \mathrm{fa}$ of the Dutch Copyright Act, indicated by the "Taverne" license above, 
Olivier Bochet

Equal-Budget Choice Equivalent Solutions in Exchange Economies

$\mathrm{RM} / 06 / 025$

METE@R

Maastricht research school of Economics of TEchnology and ORganizations

Universiteit Maastricht

Faculty of Economics and Business Administration P.O. Box 616

NL - 6200 MD Maastricht

phone : ++31433883830

fax : $\quad$ ++31433884873 



\title{
Equal-Budget Choice Equivalent Solutions in Exchange Economies* Olivier Bochet ${ }^{\dagger}$
}

\author{
May 2006
}

\begin{abstract}
Given a family of linear budget sets, an allocation is equal opportunity equivalent (Thomson, 1994) if there exists a common budget set such that each agent is indifferent between the bundle that he gets and the best bundle he can obtain in the choice set. We first study the robustness properties of equal opportunity equivalent correspondences with respect to change in preferences. We impose independence to irrelevant preference changes and connect this property with the implementation of rules via some game-theoretic solution concept. We provide an equivalence result with the equal-income Walrasian rule. Next, we study robustness with respect to change in the number of agents and derive a characterization of the equal-income Walrasian rule. Our results provide additional justifications for the equal-division of resources as a first step toward fairness.
\end{abstract}

Keywords: Fair allocations, Equal-income Walrasian solution, Equalbudget choice equivalence, Robustness, Implementability via some game theoretical solution concept.

*I thank François Maniquet for stimulating discussions on this topic. I also thank Bettina Klaus and Toyotaka Sakai for helpful comments and discussions that greatly improved this paper.

†University of Maastricht and CORE. Contact: o.bochet@algec.unimaas.nl Webpage: www.personeel.unimaas.nl/o.bochet 


\section{Introduction}

The literature on fair allocations is concerned with the division of several goods among agents with identical claims on the goods but different preferences. The model we use in this paper is that of exchange economies with a variable number $N$ of agents and $L$ infinitely divisible goods. An allocation rule that is central in this context is the equal-income Walrasian rule. Our position on fairness in this paper is that of equality of choice (Thomson, 1994). Given a family of choice sets, an allocation is fair if there exists a common choice set in that family such that each agent is indifferent between the bundle he is assigned and the best bundle he can obtain in the choice set. ${ }^{1}$ This fairness property is termed equal-opportunity equivalence. ${ }^{2}$ This notion generalizes many concepts used in the literature on fair allocations by appropriately choosing the family of choice sets $\mathcal{B}$. For instance, if $\mathcal{B}=\left\{x_{0}: x_{0} \in \mathbb{R}_{+}^{L}\right\}$, then the set of allocations that are equal-opportunity equivalent with respect to $\mathcal{B}$ is the egalitarian equivalent rule introduced by Pazner and Schmeidler (1978). Next, if $\mathcal{B}=\left\{x: x \in \mathbb{R}_{+}^{L n}\right\}$, the set of allocations that are equal-opportunity equivalent with respect to $\mathcal{B}$ is the envy-free rule introduced by Foley (1968). Families of such choice sets may be of different dimensions and are indeed numerous.

We are interested in the family of linear choice sets. Given a common reference bundle $\alpha$, a family of linear choice sets is parametrized by price vectors. An allocation rule is equal-budget choice equivalent with respect to a family of linear choice sets indexed by $\alpha$ if, for each preference profile, for each allocation $x$ that the rule selects, there exists a price vector and a choice set such that each agent is indifferent between the bundle he gets at $x$ and the best bundle he can afford in the common choice set.

Equal-budget choice equivalence combines idea of egalitarian-equivalence with that of equal-opportunity and Walrasian equilibrium. To fix ideas about the equal-budget choice equivalent allocation rules generated by such families, let $\alpha$ be the point of equal division of the aggregate endowment. Then, the equal-budget choice equivalent allocation rule generated is the equal-income

\footnotetext{
${ }^{1}$ See also Nicolò and Perea (2005).

${ }^{2}$ In addition to the notion of equal-opportunity equivalence, Thomson (1994) first introduces the notion of equal-opportunity. The former obviously gives allocation rules that, in general, selects more allocations than the latter. Moreover, the former combines the idea of equal-opportunities with that of egalitarian-equivalence.
} 
Walrasian rule. ${ }^{3}$ It has often been advocated that the point of equal division of resources is a first step towards equity and fairness. We wish to provide additional support to this claim.

We complement fairness with three robustness requirements, the first one with respect to changes in preferences and the other two with respect to changes in the number of agents when the feasible set is modified in an appropriate way. The former requirement is called independence to irrelevant preference changes. It simply states the invariance of an allocation rules with respect to change in preferences occurring only outside of the feasible set. It is a very weak property, weaker than Gevers monotonicity (see Gevers, 1986). Interestingly, this property has a clear link with the decentralization of allocation rules -i.e. their full implementation. After all, once rules satisfying desirable properties have been identified, the objective of a planner is to check whether there exist institutions-non-cooperative games-through which these allocation rules emerge as the equilibrium set of such games.

In fact, independence to irrelevant preference changes is a necessary condition for the implementation of a rule via some game-theoretic solution concept. We emphasize this connection and show that when preferences are continuous, monotonic and convex, then no allocation rule satisfies this requirement except when $\alpha$ belongs to the set of corners of the Edgeworth box. Thus, under this preference domain, the equal-income Walrasian rule is not implementable via any solution concept. However, by adding differentiability, it becomes implementable in subgame perfect equilibrium. We show that the only rule that satisfies equal-budget choice equivalence, independence to irrelevant change in preferences and neutrality is the equal-income Walrasian rule.

Next, the second robustness property we study is termed consistency (see Thomson, 1988). We show that the only rule that satisfies equal-budget choice equivalence and consistency is the equal-income Walrasian rule.

Finally, our last property is called replication invariance (see Thomson, 1988). We show that a Pareto efficient selection of an equal-budget choice equivalent rule satisfies consistency and replication invariance if and only if it is a subrule of the equal-income Walrasian rule. This result is in line with Theorem 3 in Thomson (1988).

The paper proceeds as follows. In section two, we introduce the necessary definitions and notations. Section three presents the main results and offers

\footnotetext{
${ }^{3}$ See Proposition 1 in section 3 for a formal proof of this statement.
} 
some concluding remarks.

\section{Definitions and notations}

There are $L$ infinitely divisible goods, $L \geq 2$, and a finite set of agents $N=\{1,2, \ldots, n\}$ drawn from an infinite population $\mathcal{N}$, with $n \geq 2$. The consumption set of each agent $i \in N$ is $\mathbb{R}_{+}^{L}$. For each agent $i \in N, R_{i}$ is the complete and transitive binary relation on $\mathbb{R}_{+}^{L}$ indicating (weak) preferences. The associated strict preference and indifference relations are denoted by $P_{i}$ and $I_{i}$, respectively. The set of possible preferences of each agent $i \in N$ is defined by $\mathcal{R}_{i}$. Denote by $\mathcal{R}^{N}=\prod_{i \in N} \mathcal{R}_{i}$ the set of possible preference profiles. A typical preference profile is $R=\left(R_{i}\right)_{i \in N} \in \mathcal{R}^{N}$. For each $R \in \mathcal{R}^{N}$ and each $M \subset N$, let $R_{M}=\left(R_{i}\right)_{i \in M}$.

We take as given the aggregate endowment $\bar{\omega} \gg 0 .{ }^{4}$ Once the set of agents is fixed, $\bar{\omega}$ is also fixed across preference profiles. An economy is thus simply a list of preference relations and an aggregate endowment. Formally, given $N$, an economy is a list $(R, \bar{\omega}) \in E=\left\{\mathcal{R}^{N} \times \mathbb{R}_{+}^{L}: n \in \mathbb{N}\right\}$. We consider two classes of preferences that define two domains of economies $E_{C}^{N}$ and $E_{D}^{N}{ }^{5}$

Class of preferences $\mathcal{R}_{C}^{N}$ : For each agent $i \in N$, each $R_{i} \in \mathcal{R}_{i}$ is continuous, convex and monotonic. ${ }^{6}$

Class of preferences $\mathcal{R}_{D}^{N}$ : For each agent $i \in N$, each $R_{i} \in \mathcal{R}_{i}$ is convex, monotonic and representable by a differentiable utility function.

Given an aggregate endowment $\bar{\omega}$, a (feasible) allocation is a list of bundle $\left(x_{i}\right)_{i \in N} \in \mathbb{R}_{+}^{L n}$ such that $\sum x_{i} \leq \bar{\omega}$. Given an agent $i \in N, x_{i}^{l} \in \mathbb{R}_{+}$stands for the quantity of good $l$ received by agent $i$ at bundle $x_{i}$. For any allocation $x$, and any $M \subset N$, let $x_{M}$ stand for the sub-allocation restricted to agents in $M$. Likewise, let $x_{N \backslash M}$ stand for the sub-allocation restricted to agents in $N \backslash M$.

\footnotetext{
${ }^{4}$ We order vectors with the usual conventions, $\gg,>, \geq$.

${ }^{5} R_{C}^{N}$ is the domain of classical preferences, while for $R_{D}^{N}$ is the subdomain of classical preferences that are representable by a differentiable utility function.

${ }^{6}$ A preference relation $R_{i}$ defined over $\mathbb{R}_{+}^{L}$ is convex if, for every $x_{i}$ and $y_{i} \in \mathbb{R}_{+}^{L}$ such that $x_{i} P_{i} y_{i}$, we have that $\lambda x_{i}+(1-\lambda) y_{i} P_{i} y_{i}$ for every $\lambda \in(0,1]$.

A preference relation $R_{i}$ defined over $\mathbb{R}_{+}^{L}$ is monotonic if, for each $x_{i}$ and $y_{i} \in \mathbb{R}_{+}^{L}$, $x_{i} \gg y_{i}$ implies that $x_{i} P_{i} y_{i}$.
} 
Given $\bar{\omega}$, the set of feasible allocations $A(\bar{\omega})$ is,

$$
A(\bar{\omega})=\left\{x \in \mathbb{R}_{+}^{L n}: \sum x_{i} \leq \bar{\omega}\right\} .
$$

Let $A_{i}$ be the projection of $A$ onto agent $i$ 's consumption space. Formally,

$$
A_{i}(\bar{\omega})=\left\{x_{i} \in \mathbb{R}_{+}^{L}: x_{i}^{\ell} \in\left[0, \bar{\omega}^{\ell}\right] \text { for each } \ell=1, \ldots, L\right\} .
$$

A bundle $x_{i}$ is a non-trivial corner of $A_{i}(\bar{\omega})$ if $x_{i}^{\ell}=\bar{\omega}^{\ell}$ and $x_{i}^{k}=0$ for some $\ell, k \leq L$. We say that $x_{i}$ is a trivial corner of $A_{i}$ if $x_{i}^{\ell}=\bar{\omega}^{\ell}$ for each $\ell=1, \ldots, L$ or $x_{i}^{\ell}=0$ for each $\ell=1, \ldots, L$.

An allocation $x \in A(\bar{\omega})$ is a non-trivial corner of $A(\bar{\omega})$-the Edgeworth cube - if there exists $i \in N$ such that $x_{i}$ is a non-trivial corner of $A_{i}(\bar{\omega})$. Trivial corners of $A(\bar{\omega})$ are defined in the same fashion. Let $C(A(\bar{\omega}))$ be the set of non-trivial corners of the Edgeworth cube. Abusing language, we say that a bundle $x_{i}$ is an element of $C(A(\bar{\omega}))$ if it is a non-trivial corner of $A_{i}(\bar{\omega})$. From now on, we refer to non-trivial corners simply as corners. This should cause no confusion.

Let $\Delta^{L-1}$ be the $(L-1)$-dimensional simplex. For each agent $i \in N$, denote by $B_{i}(p, \alpha)$ the budget set generated by $p \in \Delta^{L-1}$ and the bundle $\alpha \in A_{i}$.

$$
B_{i}(p, \alpha) \equiv\left\{x_{i} \in A_{i}(\bar{\omega}): p \cdot x_{i} \leq p \cdot \alpha\right\} .
$$

Given an agent $i \in N$, a preference relation $R_{i} \in \mathcal{R}_{i}$ and a bundle $x_{i} \in A_{i}(\bar{\omega})$, define $L C_{i}\left(x_{i}, R_{i}\right)=\left\{y_{i} \in A_{i}(\bar{\omega}): x_{i} R_{i} y_{i}\right\}$ to be the lower contour set at $x_{i} ; U C_{i}\left(x_{i}, R_{i}\right)=\left\{y_{i} \in A_{i}(\bar{\omega}): y_{i} R_{i} x_{i}\right\}$ the upper contour set at $x_{i} ; S L C_{i}\left(x_{i}, R_{i}\right)=\left\{y_{i} \in A_{i}(\bar{\omega}): x_{i} P_{i} y_{i}\right\}$ the strict lower contour set at $x_{i}$ and $I_{i}\left(x_{i}, R_{i}\right)=\left\{y_{i} \in A_{i}(\bar{\omega}): x_{i} I_{i} y_{i}\right\}$, the indifference curve through $x_{i}$.

We are interested in allocation rules satisfying several desirable properties. An allocation rule $f: E \rightarrow A(\bar{\omega})$ is a correspondence that associates to each preference profile a set of allocations. Let $\left.\arg \max R_{i}\right|_{B_{i}(p, \alpha)}$ be the set of maximizers of the preference relation $R_{i}$ over the set $B_{i}(p, \alpha)$.

Definition: The allocation $x \in A(\bar{\omega})$ is an equal-income Walrasian allocation for $(R, \bar{\omega}) \in E^{N}$ if there exists $p \in \Delta^{L-1}$ such that for all $i \in N$, $\left.x_{i} \in \arg \max R_{i}\right|_{B_{i}\left(p, \frac{\bar{\omega}}{n}\right)}$.

The equal-income Walrasian correspondence $W E_{E}: E^{N} \rightarrow A(\bar{\omega})$ associates to each economy $(R, \bar{\omega})$ its set of equal-income Walrasian allocations. 
Definition: Given $(R, \bar{\omega}) \in E^{N}$, the allocation $x \in A(\bar{\omega})$ is Pareto efficient if there does not exist $y \in A(\bar{\omega})$ such that $y_{i} R_{i} x_{i}$ for each $i \in N$ and $y_{j} P_{j} x_{j}$ for some $j \in N$.

Given $(R, \bar{\omega}) \in E^{N}$, an allocation $x \in A(\bar{\omega})$ is derived from a choice set $B \subset \mathbb{R}_{+}^{L}$ if for each $i \in N$ we have that $x_{i} \in B$. Define by $\mathcal{B}$ a family of choice sets.

Definition: An allocation $x \in A(\bar{\omega})$ is equal-opportunity equivalent relative to the family $\mathcal{B}$ for $(R, \bar{\omega}) \in E^{N}$ if there exists $B \in \mathcal{B}$ such that for each $i \in N, x_{i} I_{i} x_{i}^{*}$ for each $\left.x_{i}^{*} \in \arg \max R_{i}\right|_{B}$.

The family of choice sets we gave is very large. We will restrict our attention to families of linear choice sets. A family of linear choice sets is indexed by a bundle $\alpha=\alpha_{\bar{\omega}} \cdot \bar{\omega}=\left(\alpha_{\bar{\omega}_{1}} \cdot \bar{\omega}_{1}, \ldots, \alpha_{\bar{\omega}_{L}} \cdot \bar{\omega}_{L}\right)$, with $\alpha_{\bar{\omega}_{\ell}} \in[1,0]$ for each $\ell=1, \ldots, L$, and parametrized by price vectors $p \in \Delta^{L-1} .^{7}$ That is, once a point $\alpha \in \mathbb{R}_{+}^{L}$ has been fixed, the family of choice sets contains every budget set generated by $p \in \Delta^{L-1}$ and $\alpha$. More formally, given $\alpha \in \mathbb{R}_{+}^{L}$ and $p \in$ $\Delta^{L-1}$, let $B_{\alpha}(p) \equiv\left\{z \in \mathbb{R}_{+}^{L}: p \cdot z \leq p \cdot \alpha\right\}$ and $\mathcal{B}_{\alpha} \equiv\left\{B_{\alpha}(p): p \in \Delta^{L-1}\right\}$.

We are interested in several properties of allocation rules. Our first requirement is an axiom of fairness.

Equal-budget choice equivalence: A solution $f$ satisfies equal-budget choice equivalence relative to $\mathcal{B}_{\alpha}$ if,

1) For each $(R, \bar{\omega}) \in E^{N}$, each $x \in f(R, \bar{\omega})$ is equal-opportunity equivalent relative to the family $\mathcal{B}_{\alpha}$ for $(R, \bar{\omega})$.

2) There does not exist an equal-opportunity equivalent allocation y relative to $\mathcal{B}_{\alpha}$ for $(R, \bar{\omega})$ such that $y \notin f(R, \bar{\omega})$.

Given $\alpha$, let $f_{\alpha}$ be the equal-budget choice equivalent allocation rule relative to $\mathcal{B}_{\alpha}$. Our next property requires a form of robustness with respect to change in preferences. It states the invariance of a rule to changes in preferences occurring only outside of the feasible set of allocations.

Independence to irrelevant preference changes: A solution $f$ satisfies independence to irrelevant preference changes if and only if for each

\footnotetext{
${ }^{7}$ The reference point can be written as $\alpha$ or $\alpha_{\bar{\omega}} \cdot \bar{\omega}$. For convenience, we use both notations in the paper but it should not cause confusion.
} 


$$
\begin{aligned}
& (R, \bar{\omega}),\left(R^{\prime}, \bar{\omega}\right) \in E^{N} \text { and } x \in f(R, \bar{\omega}), \\
& {\left[R \neq R^{\prime} \text { and } R_{i} \cap A_{i}(\bar{\omega})=R_{i}^{\prime} \cap A_{i}(\bar{\omega}) \text { for each } i \in N\right] \Longrightarrow\left[x \in f\left(R^{\prime}, \bar{\omega}\right)\right] .}
\end{aligned}
$$

Our third property states the robustness of a rule with respect to changes in the number of agents when the feasible set is modified in an appropriate way. We define the property directly with respect to rules $f_{\alpha}$ : when a group of agents leaves the economy, not only they modify the feasible set of allocations, but they also modify the initial reference point $\alpha$.

Consistency: An allocation rule $f_{\alpha}$ satisfies consistency if and only if for each $(R, \bar{\omega}) \in E^{N}$, each $x \in f(R, \bar{\omega})$, each $M \subset N$,

$$
x_{N \backslash M} \in f_{\alpha_{\bar{\omega}} \cdot x_{N} \backslash M}\left(R \backslash R_{M}, \sum_{i \in N \backslash M} x_{i}\right) .
$$

Consistency pertains to a decreasing number of agents. An allocation rule is consistent if and only if any sub-allocation of a selected allocation is also selected in the "reduced" economy defined by the corresponding subset of agents and the set of allocations which can be considered as feasible for this subset of agents. An allocation is feasible for a subset of agents if it is possible to reach it while guaranteeing the selected consumption plans to each agent outside the considered subset of agents. Notice in the definition how the initial point $\alpha$ is also "scaled-down". Recall that $\alpha=\left(\alpha_{\bar{\omega}_{1}} \cdot \bar{\omega}_{1}, \ldots, \alpha_{\bar{\omega}_{L}} \cdot \bar{\omega}_{L}\right)$. The point $\alpha_{\bar{\omega}} \cdot \bar{\omega}_{N \backslash M}$ is,

$$
\alpha_{\bar{\omega}} \cdot \bar{\omega}_{N \backslash M}=\left(\alpha_{\bar{\omega}_{1}} \cdot\left(\bar{\omega}_{1}-x_{M}^{1}\right), \ldots, \alpha_{\bar{\omega}_{L}} \cdot\left(\bar{\omega}_{L}-x_{M}^{L}\right)\right) .
$$

We complement consistency with a robustness axiom that applies to situations in which the number of agents increases, and when the feasible set is modified in an appropriate way. Thomson (1988) defines four robustness properties of rules with respect to an increase in the number agents. Among these, the one that has been the object of most of the attention in the literature is replication invariance that we define next.

Replication invariance: An allocation rule $f_{\alpha}$ satisfies replication invariance if and only if for each $(R, \bar{\omega}) \in E^{N}$, each $x \in f(R, \bar{\omega})$ and each $\lambda \in \mathbb{N}$,

$$
\lambda x \in f_{\alpha_{\bar{\omega}} \cdot \lambda \bar{\omega}}(\lambda R, \lambda \bar{\omega}) .
$$


Apart from fairness and robustness, a central authority-e.g. a planneris interested in the possibility of obtaining the allocations selected by the rule as equilibrium outcomes of some non-cooperative game-i.e. through an institution. Our point of view here is that decentralization entails full implementation of a solution. Before introducing the property of implementability via some game-theoretic solution concept, let us add some necessary definitions.

A game form or mechanism is a game tree with possibly simultaneous moves. ${ }^{8}$ More formally, it is defined as an array $\Gamma=(N, T, g)$ where $N$ is the set of players, $T$ a game tree, and $g$ is an outcome function that associates a feasible allocation with each path of play. The set of nodes of the tree $T$ is denoted $S$. The initial node is $s_{0}$. The set of terminal nodes of the tree $T$ is denoted $Z$. Let $M_{i}$ be the set of strategies of player $i$, and let $M_{i}^{s}$ denote the set of strategies available to player $i$ at node $s$. Denote by $M=\prod_{i} M_{i}$, the set of strategy profiles. Suppose the strategy profile $m \in M$ is played. Let $g(m)_{i}$ stand for bundle obtained by agent $i \in N$ at the allocation prescribed by the path induced by $m$, that is, $g(m)$. Let $g(m, s)$ denotes the outcome corresponding to $m$ starting at node $s$. As is common in the implementation literature, we confine our attention to pure strategies.

For the definitions at hand, suppose that the only characteristics that can vary are the preferences of agents. That is, the set of agents $N$ and the endowment $\bar{\omega}$ are fixed. An economy then reduces to a preference profile $R \in \mathcal{R}^{N}$. Given an economy $R \in \mathcal{R}^{N}$, the mechanism $\Gamma$ defines a noncooperative game in extensive game form $(\Gamma, R)$.

Let $\mathcal{S C}$ be a game theoretic solution concept. A solution concept describes a set of predictions on how a game will be played, as a function of the agents' preferences. For each $R \in \mathcal{R}^{N}$, the set of $\mathcal{S C}$-equilibrium outcomes of $(\Gamma, R)$ is denoted $\operatorname{ESC}(\Gamma, R)$.

A solution $f$ is implementable in $\mathcal{S C}$-equilibrium if and only if for each $R \in \mathcal{R}^{N}, E \mathcal{S C}(\Gamma, R)=f(R)$.

Examples of solutions concepts are numerous. To define just a few, a Nash equilibrium of $(\Gamma, R)$ is a strategy profile $m^{*} \in M$ such that for each

\footnotetext{
${ }^{8}$ Here, we consider static as well as sequential game forms. Since a static game form can always be represented as a sequential game form with simultaneous moves, we choose this more general definition that encompasses both cases.
} 
$i \in N$

$$
g\left(m^{*}\right)_{i} R_{i} g\left(m_{i}, m_{-i}^{*}\right)_{i} \text { for each } m_{i} \in M_{i}
$$

A subgame perfect equilibrium of $(\Gamma, R)$ is a strategy profile $m^{*} \in M$ such that for all $s \in S \backslash Z$ and for all $i \in N$,

$$
g\left(m^{*}, s\right)_{i} R_{i} g\left(m_{i}, m_{-i}^{*}, s\right)_{i} \text { for each } m_{i} \in M_{i}
$$

But solutions concepts are not only limited to Nash equilibrium and its refinements. Solution concepts such as dominant strategies or undominated strategies are also part of our general definition. ${ }^{9}$

Implementability via some game-theoretic solution concept: A solution $f$ satisfies implementability via some game-theoretic solution concept if and only if there exists a game theoretic solution concept $\mathcal{S C}$ and a game form $\Gamma$ such that $f$ is implementable by $\Gamma$ via $\mathcal{S C}$.

We close this section and give the definitions of Maskin monotonicity (Maskin, 1999), a necessary condition for Nash implementation and Unchanged contour independence (see Maniquet, 2003), a sufficient condition for subgame perfect implementation. ${ }^{10}$

Maskin monotonicity: An allocation rule $f$ is Maskin monotonic if and only for each $(R, \bar{\omega}),\left(R^{\prime}, \bar{\omega}\right) \in E^{N}$, and $x \in f(R, \bar{\omega})$,

$$
\left[L C_{i}\left(R_{i}, x_{i}\right) \subseteq L C_{i}\left(R_{i}^{\prime}, x_{i}\right) \text { for each } i \in N\right] \Longrightarrow\left[x \in f\left(R^{\prime}, \bar{\omega}\right)\right] .
$$

Unchanged contour independence: A SCC $f$ satisfies unchanged contour independence if and only if for each $(R, \bar{\omega}),\left(R^{\prime}, \bar{\omega}\right) \in E^{N}$ and $x \in$ $f(R, \bar{\omega})$

$\left[I_{i}\left(R_{i}, x_{i}\right) \cap A_{i}(\bar{\omega})=I_{i}\left(R_{i}^{\prime}, x_{i}\right) \cap A_{i}(\bar{\omega})\right.$ for each $\left.i \in N\right] \Longrightarrow\left[x \in f\left(R^{\prime}, \bar{\omega}\right)\right]$.

\footnotetext{
${ }^{9}$ Our definition of solution concepts should be seen as encompassing any solution concepts commonly used in the implementation literature since they all share a common feature that will be explained in the next section: in order to break a equilibrium when going from one preference profile to another, a change in preferences is needed.

${ }^{10}$ Bochet (2005b), also shows that if non-degenerate lotteries are permitted out of equilibrium, then Maskin monotonicity and Nash implementation coincide.
} 


\section{Properties of equal-budget choice equiva- lent rules}

In this section, we study equal-budget choice equivalent allocation rules. Allocation rules are indexed by a number $\alpha$ and can be decomposed into three categories that are specific to the region in which $\alpha$ is located. The first case is when ${ }^{11}$,

$$
\alpha \in\left\{0<\alpha^{\prime}<\bar{\omega}: \alpha^{\prime} \notin C(A(\bar{\omega})) \text { and } \alpha^{\prime} \neq \frac{\bar{\omega}}{n}\right\}
$$

The second case is when ${ }^{12}$,

$$
\alpha \in C(A(\bar{\omega}))
$$

Finally, the last case is when

$$
\alpha=\frac{\bar{\omega}}{n} .
$$

When $\alpha$ is the point of equal-division, $f_{\alpha}$ is equal-budget choice equivalent if and only if it coincides with the equal-income Walrasian rule. We show this result in the lemma below.

Lemma 1 (Thomson, 1994) Let the domain of preferences be $\mathcal{R}_{C}^{N}$. Consider the family of linear choice sets $\mathcal{B}_{\underline{\underline{\omega}}}$. An allocation rule $f_{\alpha}$ is equal-budget choice equivalent if and only if $f_{\alpha}=\stackrel{n}{W}{ }_{E Q}$.

Proof: Let $f$ be the equal-budget choice equivalent allocation rule with respect to $\mathcal{B}_{\frac{\omega}{n}}$ and let $R, \bar{\omega}$ and $x \in f(R, \bar{\omega})$ be given. By definition, there exists $p \in \Delta^{L-1}$ such that for each $i \in N, x_{i} I_{i} x_{i}^{*}$ for each $\left.x_{i}^{*} \in \arg \max R_{i}\right|_{B_{\frac{\omega}{n}}(p)}$. Our claim is that $p \cdot x_{i}=p \cdot \alpha$ for each $i \in N$, i.e. $\left.x_{i} \in \arg \max R_{i}\right|_{B_{\frac{\bar{\omega}}{n}}(p)}{ }^{n}$. To see this, observe first that by definition, we have that $p \cdot x_{i} \geq p \cdot x_{i}^{*}$. Suppose, contrary to our claim that for some $i \in N, p \cdot x_{i}>p \cdot x_{i}^{*}$. Summing across agents, we obtain that $\sum p \cdot x_{i}>\sum p \cdot x_{i}^{*}=p \cdot \bar{\omega}$, a contradiction with the feasibility of $x$ as an allocation. Therefore, $f \subset W_{E Q}$. The other inclusion is obvious.

Q.E.D.

\footnotetext{
${ }^{11}$ We point out that for any $\alpha \gg \frac{\bar{\omega}}{n}$, the allocation rule $f$ is empty at each preference profile.

${ }^{12}$ We voluntarily exclude the trivial corners $\{0\}$ and $\{\bar{\omega}\}$ as point of division since they both deliver empty solutions.
} 


\subsection{Robustness with Respect to Changes in Prefer- ences and Implementation}

We start this section by showing a clear connection between independence to irrelevant preference changes and the decentralization of a rule. In fact, such a robustness property is the most basic requirement for an allocation rule to be implementable via some game-theoretic solution concept. In this section, we fix the set of agents $N$ and the endowment $\bar{\omega}$. A rule $f$ is then a mapping $f: \mathcal{R}^{N} \rightarrow A(\bar{\omega})$. An economy is thus simply a preference profile $R$ and a class of economy is simply a set of preference profiles $\mathcal{R}^{N}$.

Proposition 1: A solution $f$ satisfies implementability via some gametheoretic solution concept only if it satisfies independence to irrelevant preference changes.

Proof: Since $f$ satisfies implementability via some game-theoretic solution concept, there exists a solution concept $\mathcal{S C}$ and a game form $\Gamma$ such that $f$ is implemented by $\Gamma$ via $\mathcal{S C}$. Fix a preference profile $R \in \mathcal{R}^{N}$ and $x \in f(R)$. By implementability via some game-theoretical solution concept, there exists $m \in M$ that is an $\mathcal{S C}$-equilibrium such that $g(m)=x \in \operatorname{ESC}(\Gamma, R)$.

Consider a change in preferences when going from $R$ to $R^{\prime}$ with $R_{i} \cap A_{i}=$ $R_{i}^{\prime} \cap A_{i}$ for each $i \in N$. Thus, for some $j \in N, R_{j} \neq R_{j}^{\prime}$ but for each $i \in N$, $R_{i} \cap A_{i}=R_{i}^{\prime} \cap A_{i}$. The region where preferences determined that $m$ was an $\mathcal{S C}$-equilibrium, and that $x \in \operatorname{ESC}(\Gamma, R)$, still contains the exact same set of preferences as in $R$. Therefore, if $m$ was an $\mathcal{S C}$-equilibrium under $R$, it remains one under $R^{\prime}$. As a consequence, we have that $x \in \operatorname{ESC}\left(\Gamma, R^{\prime}\right)$.

Q.E.D.

Proposition 1 states an obvious requirement for implementability via some game theoretic solution concept. Information on preferences outside of the feasible set cannot be used. The reason is that in order to break an equilibrium when going from one preference profile to another, a change in preferences is needed. For implementation via Nash equilibrium and any of its refinement, specific preference reversals are needed. For instance, implementation via Nash equilibrium requires that if $x \in f(R) \backslash f\left(R^{\prime}\right)$, there exists $i \in N$ and $y_{i} \in A_{i}$ such that $x_{i} R_{i} y_{i}$ and $y_{i} P_{i}^{\prime} x_{i}$. On the other hand, for implementation via undominated strategies, if $x \in f(R) \backslash f\left(R^{\prime}\right)$ there exists $i \in N$ and $y_{i} \in A_{i}$ such that either $x_{i} R_{i} y_{i}$ and $y_{i} P_{i}^{\prime} x_{i}$, or $x_{i} P_{i} y_{i}$ and $x_{i} I_{i}^{\prime}$ 
$y_{i} \cdot{ }^{13}$ Finally, implementation via subgame perfect equilibrium requires that if $x \in f(R) \backslash f\left(R^{\prime}\right)$, there exists $i \in N$, and $y_{i}, z_{i} \in A_{i}$ such that $y_{i} R_{i} z_{i}$ and $z_{i} P_{i} x_{i}$.

However preference changes outside of the feasible set cannot be tested since they occur at allocations that are not feasible. Therefore, an allocation rule sensitive to change in preferences outside of the feasible set can never be implemented, and this whatever solution concept used. ${ }^{14}$

Next we show that when the domain is $\mathcal{R}_{C}^{N}$, the only equal-budget choice equivalent rules that are not sensitive to a change in preferences occuring only outside of the feasible set are the rules $f_{\alpha}$ with $\alpha \in C(A(\bar{\omega}))$. Differentiability plays a crucial rule in the implementation of the equal-income Walrasian rule. When indifference curves can have kinks, the equal-income Walrasian rule is not implementable via any solution concept. As expected, the problem comes from allocations that are at the boundary of the feasible set but it goes beyond the classical impossibility result on the Nash implementation of this rule.

Proposition 2: Suppose the class of preferences is $\mathcal{R}_{C}^{N}$. An equal-budget choice equivalent allocation rule $f_{\alpha}$ satisfies implementability if and only if $\alpha \in C(A(\bar{\omega})) . \alpha \in C(A(\bar{\omega}))$.

Proof: The proof relies on a series of lemmas.

Lemma 1: Suppose that,

$$
\alpha \in\left\{0<\alpha^{\prime}<\bar{\omega}: \alpha^{\prime} \notin C(A(\bar{\omega})) \text { and } \alpha^{\prime} \neq \frac{\bar{\omega}}{n}\right\} .
$$

The equal-budget choice equivalent allocation rule $f_{\alpha}$ with respect to $\mathcal{B}_{\alpha}$ does not satisfy implementability.

Proof: We use counter-examples to prove the claim. The examples we construct are for $\alpha=\frac{\bar{\omega}}{K}$ for $K>n .{ }^{15}$ The proof generalizes to any

$$
\alpha \in\left\{0<\alpha^{\prime}<\bar{\omega}: \alpha^{\prime} \notin C(A(\bar{\omega})) \text { and } \alpha^{\prime} \neq \frac{\bar{\omega}}{n}\right\} \text {. }
$$

\footnotetext{
${ }^{13}$ See Jackson (1992) for a definition of implementation in undominated strategies.

${ }^{14}$ Notice also that such allocations rules cannot be virtually implemented either. For a definition of Virtual Nash implementation, see for instance Abreu and Sen (2001) or Bochet and Maniquet (2005).

${ }^{15}$ The case $K<n$ is trivial. For any $\alpha \gg \frac{\bar{\omega}}{n}$, the equal-budget choice equivalent allocation rule with respect to $\mathcal{B}_{\alpha}$ is then empty at every preference profiles. When $\alpha>\frac{\bar{\omega}}{n}$, this is no longer true but the same type of examples we construct in the lemma can be applied.
} 


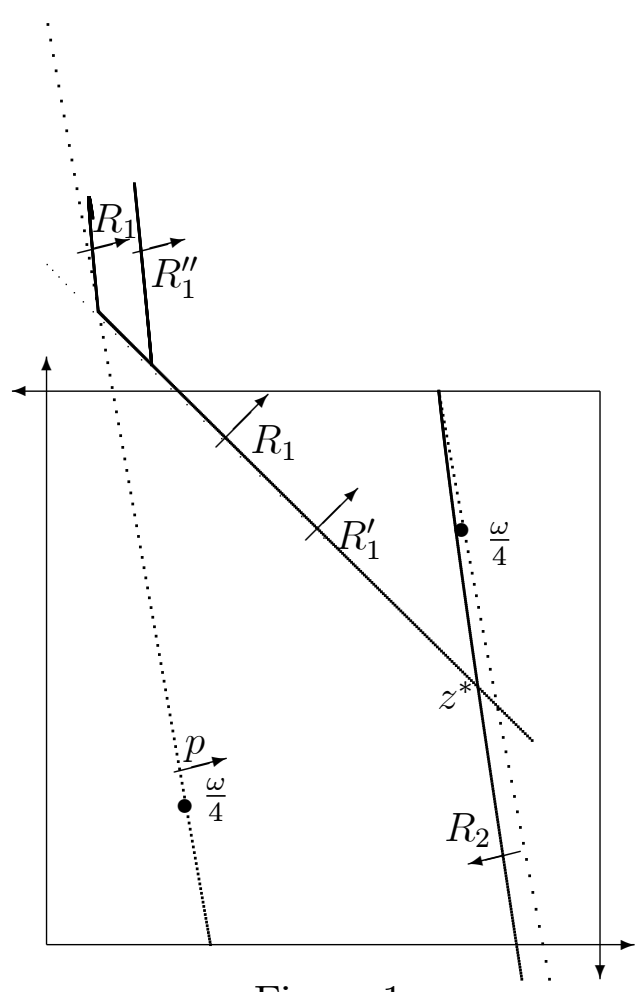

Figure 1

We consider two agents-two goods exchange economy with two possible preference profiles $R=\left(R_{1}, R_{2}\right)$ and $R^{\prime}=\left(R_{1}^{\prime}, R_{2}\right)$. The preferences are represented by utility functions as follows.

$u_{1}\left(\cdot, R_{1}\right)=\min \left\{x_{1}+y_{1} ; x_{1}+\frac{1}{K+1} y_{1}+\frac{5 K}{K+1}\right\}$

$u_{1}\left(\cdot, R_{1}^{\prime}\right)=x_{1}+y_{1}$

$u_{2}(\cdot)=(K+1) x_{2}+y_{2}$

The endowment is $\bar{\omega}=(4,4)$.

For each $K>n$, when the profile is $R$, there exists an allocation $z^{*} \in$ $f_{\frac{\bar{\omega}}{K}}(R)$, that is equal-budget choice equivalent and for which $\left.\left(x_{1}, 5\right) \in \arg \max R_{1}\right|_{B_{\frac{\bar{\omega}}{K}}(p)} \notin$ $A_{i}, x_{1}>0$, for some $i \in N$ and $p=(K, 1)$. The situation is depicted for the case $K=4$ in Figure 1 above.

There is an equal-budget choice equivalent allocation,

$$
z^{*}=\left(\left(\frac{123}{48}, \frac{137}{48}\right),\left(\frac{69}{48}, \frac{55}{48}\right)\right),
$$

and $\left.\arg \max R_{1}\right|_{B_{\overline{\frac{\omega}{4}}}(p)}=\left(\frac{5}{12}, 5\right)$. However, allocation $z^{*}$ is no longer equalbudget choice equivalent with respect to $B_{\frac{\bar{\omega}}{4}}(p)$ at $R^{\prime}$ : the indifference curve of agent 1 at the point $\left(\frac{5}{12}, 5\right)$ extends below $B_{\frac{\omega}{4}}(p)$ and hence $\left(\frac{5}{12}, 5\right) \notin$ $\left.\arg \max R_{1}^{\prime}\right|_{B_{\overline{\frac{\omega}{4}}}(p)}=\left(0, \frac{20}{3}\right)$. Finally, observe that the preferences of agents 
inside the feasible set have not changed. The correspondence $f_{\frac{\bar{\omega}}{4}}$ is not implementable in any game theoretical solution concept. It is clear that the same conclusion applies to any $K>n$.

The counter-examples we used were based on an extension of an indifference curve below the set $B_{\frac{\omega}{K}}(p)$ for agent 1 when going from $R$ to $R^{\prime}$. For each $K>n$, the same conclusion remains true even if there is an expansion of the lower contour set at $z^{*}$ outside of the feasible set. To see this, suppose that there is also a third preference profile $\left(R_{1}^{\prime \prime}, R_{2}\right)$ at which preferences of agent 1 are given by $u_{1}\left(\cdot, R_{1}^{\prime \prime}\right)=\min \left\{x_{1}+y_{1} ; x_{1}+\frac{1}{K+1} y_{1}+\frac{4.5 K}{K+1}\right\}$. As seen in Figure 1, there is an expansion of the lower contour set for agent 1 when going from $R$ to $R^{\prime \prime}$ but the expansion occurs outside of the feasible set while the preferences inside the feasible set remain unchanged. This shows that the violation of implementability is not restricted to $\mathcal{R}_{C}^{N}$ but also applies to $\mathcal{R}_{D}^{N}$ as well.

Finally, notice that the examples we have used are based on extension of budget sets beyond the upper boundary of the Edgeworth box. For a point $\alpha=(0, k)$ with $k<\bar{\omega}^{2}$, the extension of the budget sets will occur on the right boundary of the box. The same examples as we have constructed above can be adapted to this case.

Q.E.D.

Lemma 2: Suppose the class of preferences is $\mathcal{R}_{C}^{N}$. The allocation rule $W_{E Q}$ does not satisfy implementability

Proof: We adapt the counter example of lemma 1 to the case $K=n=2$. The aggregate endowment is $\bar{\omega}=(4,4)$. The preferences of agents are as follows.

$u_{1}\left(\cdot, R_{1}\right)=\min \left\{x_{1}+y_{1} ; x_{1}+\frac{1}{3} y_{1}+\frac{8}{3}\right\}$ and $u_{1}\left(\cdot, R_{1}^{\prime}\right)=x_{1}+y_{1}$.

$u_{2}(\cdot)=2 x_{2}+y_{2}$

When the profile is $R$, there exists an equal-income Walrasian equilibrium $\left(z^{*}, p^{*}\right)$ on the boundary of the feasible set with $z^{*}=((1,4) ;(3,0))$ and $p^{*}=(2,1)$.

The situation is depicted graphically in Figure 2 on the next page.

Allocation $z^{*}$ is no longer an equal-income Walrasian equilibrium under $R^{\prime}$. However, there exists no agent $i$ and no pair $x_{i}, y_{i} \in A_{i}$ such that

$$
x_{i} R_{i} y_{i} \text { and } y_{i} P_{i}^{\prime} x_{i} \text {. }
$$




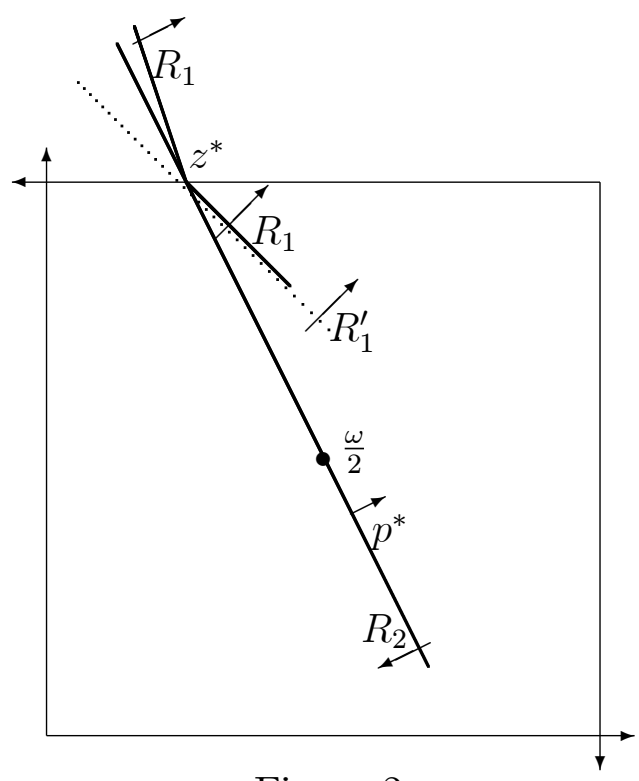

Figure 2

In conclusion, the equal-income Walrasian rule is not implementable in any solution concept. ${ }^{16}$

Q.E.D.

Lemma 3: Suppose that $\alpha \in C(A(\bar{\omega}))$, then $f_{\alpha}$ satisfies implementability. Proof: Clearly, $f_{\alpha}$ satisfies independence to irrelevant preference changes. To see this, observe that whenever the common budget set extends outside of the feasible set, then indifference curves of agents never intersect. That is, whenever given $p \in \Delta^{L-1}$, the common budget set extends outside of the feasible set, we have that $\cap_{i \in N}\left\{x_{i} \in B_{\alpha}(p)\right\} \neq \emptyset$.

Though necessary, this condition is far from being sufficient. In fact, it is enough to observe that for any such $\alpha, f_{\alpha}$ satisfies unchanged contour independence, a sufficient condition for subgame perfect implementation. ${ }^{17}$ Given $R \in \mathcal{R}_{C}^{N}$,for each $x \in f_{\alpha}(R)$, there exists $p \in \Delta^{L-1}$ such that $\cap_{i \in N}\left\{x_{i} \in B_{\alpha}(p)\right\}=\emptyset$, and for each $i \in N,\left.x_{i} I_{i} \arg \max R_{i}\right|_{B_{\alpha}(p)} \in A_{i}$. Consider a profile $R^{\prime} \in \mathcal{R}_{C}^{N}$ such that $I_{i}\left(R_{i}, x_{i}\right) \cap A_{i}=I_{i}\left(R_{i}^{\prime}, x_{i}\right) \cap A_{i}$ for each $i \in N$. We have that for each $i \in N,\left.z_{i} \in \arg \max R_{i}\right|_{B_{\alpha}(p)} \Longleftrightarrow$

\footnotetext{
${ }^{16}$ Obviously, the same is true for any initial distribution of the aggregate endowment different of equal-division: if the domain is $\mathcal{R}^{N D}$, then the Walrasian correspondence is not implementable in any solution concept (see Bochet, 2005a).

${ }^{17}$ Observe that if $\alpha \in C(A(\bar{\omega})), f_{\alpha}$ is not Maskin monotonic and hence not Nash implementable.
} 
$\left.z_{i} \arg \max R_{i}^{\prime}\right|_{B_{\alpha}(p)}$. Therefore, $x \in f\left(R^{\prime}\right)$.

Q.E.D.

The combination of lemma 1 to 3 completes the proof of Proposition 2.

Proposition 3: Suppose the class of preferences is $\mathcal{R}_{D}^{N}$. An equal-budget choice equivalent allocation rule $f_{\alpha}$ satisfies implementability if and only if $\alpha \in C(A(\bar{\omega})) \cup\left\{\frac{\bar{\omega}}{n}\right\}$.

Proof: We have already shown in lemma 1 that even if preferences are differentiable and $\alpha \in\left\{0<\alpha^{\prime}<\bar{\omega}: \alpha^{\prime} \notin C(A(\bar{\omega}))\right.$ and $\left.\alpha^{\prime} \neq \frac{\bar{\omega}}{n}\right\}$, then $f_{\alpha}$ cannot satisfy equal-budget choice equivalence and implementability.

Finally, observe that $W_{E Q}$ now satisfies unchanged contour independence (see Maniquet (2003) and Bochet (2005a)). ${ }^{18}$

Q.E.D.

Remark 1: Only a few allocation rules satisfy equal-budget choice equivalence and implementability. By the first welfare theorem, $W_{E Q}$ only contains Pareto efficient allocations. This is clearly not the case when $\alpha \neq \frac{\bar{\omega}}{n}$. We point out in passing that whenever $\alpha \neq \frac{\bar{\omega}}{n}$, there does not exist a Pareto efficient and Nash implementable selection of the equal-budget choice equivalent allocation rule $f_{\alpha}$.

Moreover, our point of view is that the boundary rules do not offer a sensible approach to fairness. After all, when deciding how to first divide the resources, the preferences of agents are in general unknown to the planner. By discarding some goods, the planner goes against a principle of neutrality with respect to goods. For instance suppose that there are two goods, food and water. The planner should not decide to give water to agents but no food to start with when both items are available. It implies that the name of the goods should not matter. This is the familiar neutrality axiom defined below.

Neutrality: An allocation rule $f_{\alpha}$ satisfies neutrality if and only if for each permutation $\pi: L \rightarrow L$ and each $R \in \mathcal{R}^{N}$,

$$
x \in f_{\alpha}(R) \Longrightarrow \pi \circ x \in f_{\pi \circ \alpha}(\pi \circ R) .
$$

Based on this argument, a strengthening of proposition 3 with neutrality would provide a simple "characterization" of the equal-income Walrasian

\footnotetext{
${ }^{18}$ Obviously, the equal-income Walrasian allocation rule is not implementable in dominant strategies, in undominated strategies or in Nash equilibrium.
} 
rule. In addition, it provides a further justification of equal-division of resources as a first step towards fairness.

\subsection{Robustness with Respect to Change in the Num- ber of Agents}

In this section we go back to the initial setting introduced in section 2, where the number of agents in the economy can vary. We start with the study of our second robustness property, consistency. ${ }^{19}$

Proposition 4: Suppose the class of economies is $E_{D}^{N}$. An allocation rule $f_{\alpha}$ satisfies consistency if and only if $\alpha=\frac{\bar{\omega}}{n}$.

Proof:

First part: The fact that $W_{E Q}$ satisfies consistency is well-known (see Thomson, 1988). We recall its proof for the sake of completeness.

Take an economy $(R, \bar{\omega}) \in E_{D}^{N}$ and fix $x \in W_{E Q}(R, \bar{\omega})$. By definition, there exists $p \in \Delta^{L-1}$ such that for each $i \in N,\left.x_{i} \in \arg \max R_{i}\right|_{B_{\frac{\bar{\omega}}{n}}(p)}$. Given the assumptions on preferences, for each $i \in N, p \cdot x_{i}=p \cdot \frac{\bar{\omega}}{n}$. Consider a subeconomy $M \subset N$. The total resources available in this subeconomy after the departure of agents in $N \backslash M$ with their respective bundle is $\sum_{i \in M} x_{i}$. Hence, allocation $x_{M}$ is still feasible. Since $p \cdot x_{i}=p \cdot \frac{\bar{\omega}}{n}$, we have that $p \cdot \frac{\sum_{j \in M} x_{j}}{|M|}=p \cdot \frac{\bar{\omega}}{n}$. Therefore, $B_{\frac{\bar{\omega}}{n}}(p)=B_{\frac{\sum_{j \in M} x_{j}}{|M|}}(p)$ and thus $x_{j} \in f_{\frac{\sum_{j \in M} x_{j}}{|M|}}\left(R \backslash R_{N \backslash M}, \quad \sum_{j \in M} x_{j}\right)$.

Second part: To see that when $\alpha$ is one of the corners of the Edgeworth cube, consistency may be violated, let us look at the following example.

There are three agents and two goods, $\bar{\omega}=(4,2)$. At profile $R \in$ $\mathcal{R}_{D}^{N}$, agents 1 and 2 have utility functions given by $u_{i}\left(x_{i}, y_{i}\right)=\frac{2}{3} x_{i}+y_{i}$ and $u_{3}\left(x_{3}, y_{3}\right)=3 x_{3}+y_{3}$. Consider $\alpha=\left(0, \bar{\omega}_{y}\right)$ and the allocation $z=$ $((1.5,1) ;(1.5,1) ;(1,0))$. This allocation is equal-opportunity equivalent. Given $p=(2,1),\left.\arg \max R_{i}\right|_{B_{\alpha}(p)}=(0,2)$ for $i=1,2,\left.\arg \max R_{3}\right|_{B_{\alpha}(p)}=(1,0)$ and $\left.\arg \max R_{j}\right|_{B_{\alpha}(p)} I_{i} z_{j}$ for each $j=1,2,3$. Suppose that agent 1 leaves the economy with $z_{1}$. By consistency, $((1.5,1) ;(1,0)) \in f_{(0,1)}\left(R \backslash R_{1},(2.5,1)\right)$.

\footnotetext{
${ }^{19}$ Thomson (1988) showed that if indifference curves have kinks, then $W_{E Q}$ does not satisfy consistency. Hence, for the remainder of the paper, the class of economies we consider is $E_{D}^{N}$.
} 
The only price vector such that $\left.\arg \max R_{3}\right|_{B_{(0,1)}(p)}=(1,0)$ is $p=(1,1)$. At this prices, $\left.\arg \max R_{2}\right|_{B_{(0,1)}(p)}=(0,1)$ but $\left.z_{2} P_{i} \arg \max R_{2}\right|_{B_{(0,1)}(p)}$, a contradiction with the fact that $f_{\alpha}$ satisfies equal-budget choice equivalence.

Third part: Next, we show that when $\alpha \notin C(A(\bar{\omega})) \cup\left\{\frac{\bar{\omega}}{n}\right\}$, consistency is also violated. We use one counter-example but it should be clear that the same can be shown for any other $\alpha \notin C(A(\bar{\omega})) \cup\left\{\frac{\bar{\omega}}{n}\right\}$.

Again we consider a three agents-two goods example with $\bar{\omega}=(5,2)$ and $\alpha=(0.5,2)$. At profile $R \in \mathcal{R}_{D}^{N}$, agents $i=1,2$ have utility functions given by $u_{i}=x_{i}+y_{i}$ and $u_{3}=\left(x_{3}\right)^{2} y_{3}$. Allocation $z=((2,0.5) ;(2,0.5) ;(1,1))$ is equal-opportunity equivalent with respect to $\mathcal{B}_{\alpha}$ for $p=(2,1)$. Suppose now that agent 1 leaves with $z_{1}$. The reduced economy has $\bar{\omega}=(3,1.5)$ and $\alpha=(0.3,1.5)$. By consistency, $((2,0.5) ;(1,1)) \in f_{(0.3,1.5)}\left(R \backslash R_{1},(3,1.5)\right)$. The only price vector such that $\left.\arg \max R_{2}\right|_{B_{(0.3,1.5)}(p)} I_{2}(2,0.5)$ is $p=\left(\frac{15}{22}, 1\right)$. However, $\left.\arg \max R_{3}\right|_{B_{(0.3,1.5)}\left(\frac{15}{2}, 1\right)} P_{3} z_{3}$, a contradiction with equal-budget choice equivalence.

We conclude that the proposition holds if and only if $\alpha=\frac{\bar{\omega}}{n}$. Q.E.D.

We conclude our results by the study of replication invariance. We provide a characterization that parallels a result that appears in Thomson (1988). He shows that a selection from the Pareto efficient and equal-split lower bound correspondence satisfies consistency and replication invariance if and only if it is a subrule of the equal-income Walrasian rule.

Proposition 5: Suppose the class of preferences is $\mathcal{R}_{D}^{N}$ and fix $\alpha \in \mathbb{R}_{+}^{L}$. Any Pareto efficient selection from $f_{\alpha}$ satisfies consistency and replication invariance if and only if $\alpha=\frac{\bar{\omega}}{n}$.

Proof: Let $\alpha=\alpha_{\bar{\omega}} \cdot \bar{\omega} \in \mathbb{R}_{+}^{L},(R, \bar{\omega}) \in E_{D}^{N}$ and $x \in f_{\alpha_{\bar{\omega}} \cdot \bar{\omega}}(R, \bar{\omega})$ be given. By Pareto efficiency, there exists $p \in \Delta^{L-1}$ that supports $x$. Suppose that $x \notin W_{E Q}(R, \bar{\omega})$. Then there exists $i \in N$ with $p \cdot x_{i}>p \cdot \frac{\bar{\omega}}{n}$ and $j \in N$ with $p \cdot x_{j}<p \cdot \frac{\bar{\omega}}{n}$. By convexity and monotonicity of preferences, there exists $\lambda_{i}, \lambda_{j} \in \mathbb{N}$ and $y$ such that $y P_{j} x_{j}$, and

$$
y=\frac{\lambda_{i} x_{i}+\lambda_{j} x_{j}}{\lambda_{i}+\lambda_{j}} .
$$

Define $\lambda \equiv \max \left\{\lambda_{i}, \lambda_{j}\right\}$. By replication invariance, $\lambda x \in f_{\alpha_{\bar{\omega}} \cdot \lambda \bar{\omega}}(\lambda R, \lambda \bar{\omega})$. Consider a subeconomy of $\lambda R$ composed of $\lambda_{i}$ agents of type $i$ and $\lambda_{j}$ agents of 
type $j$. By consistency, we obtain that $\left(\lambda x_{i}, \lambda x_{j}\right) \in f_{\alpha_{\bar{\omega}}\left(\lambda_{i}+\lambda_{j}\right) \lambda y}\left(\lambda_{i} R_{i}, \lambda_{j} R_{j}, \lambda\left(\lambda_{i}+\lambda_{j}\right) y\right)$. By equal-opportunity equivalence, there exists $p \in \Delta^{L-1}$ such that $\lambda x_{k} I_{k}$ $\left.\arg \max R_{k}\right|_{B_{\lambda y}(p)} R_{k} \alpha_{\bar{\omega}}\left(\lambda_{i}+\lambda_{j}\right) \lambda y$ for $k=i, j$. This implies that $\lambda x_{j} R_{j}$ $\alpha_{\bar{\omega}}\left(\lambda_{i}+\lambda_{j}\right) \lambda y$, and $\alpha_{\bar{\omega}}\left(\lambda_{i}+\lambda_{j}\right) \lambda x_{j} P_{j} \alpha_{\bar{\omega}}\left(\lambda_{i}+\lambda_{j}\right) \lambda y$, a contradiction with the initial assumption that $y P_{j} x_{j}$.

Q.E.D.

We conclude the paper with two remarks.

Remark 2: As seen in lemma 2, for some preference domains, the equal-income Walrasian correspondence is not implementable via any gametheoretical solution concept. On the other hand, a correspondence called the constrained equal-income Walrasian correspondence, $C W_{E Q}$, is Nash implementable. Given the deficits of the allocation rules we have studied, one can alternatively look at a constrained version of equal-budget choice equivalence.

Given $\alpha \in\left\{0<\alpha^{\prime}<\bar{\omega}: \alpha^{\prime} \notin C(A(\bar{\omega}))\right.$ and $\left.\alpha^{\prime} \neq \frac{\bar{\omega}}{n}\right\}$ and $p \in \Delta^{L-1}$, a constrained linear choice set is $\bar{B}_{\alpha}(p) \equiv\left\{x \in A_{i}(\bar{\omega}): p \cdot x \leq p \cdot \alpha\right\}$. Based on this definition, we can reformulate our axiom in term of constrained equalopportunity equivalence and constrained equal-budget choice equivalence.

Constrained equal-opportunity equivalence: An allocation $x \in$ $A(\bar{\omega})$ is constrained equal-opportunity equivalent relative to the family $\mathcal{B}_{\alpha}$ for $R \in \mathcal{R}^{N}$ if there exists $\bar{B}_{\alpha}(p) \in \mathcal{B}_{\alpha}$ such that for each $i \in N, x_{i} I_{i} x_{i}^{*}$ for some $\left.x_{i}^{*} \in \arg \max R_{i}\right|_{\bar{B}_{\alpha}(p)}$.

The definition of equal-budget choice equivalence allocation rule $\bar{f}_{\alpha}$ then just follows. When $\alpha \in\left\{0<\alpha^{\prime}<\bar{\omega}: \alpha^{\prime} \notin C(A(\bar{\omega}))\right.$ and $\left.\alpha^{\prime} \neq \frac{\bar{\omega}}{n}\right\}$, every $\bar{f}_{\alpha}$ is subgame perfect implementable. However, notice that the only Nash implementable allocation rule is the constrained equal-income Walrasian rule $C W_{E Q} \cdot{ }^{20}$ Therefore, an allocation rule $\bar{f}_{\alpha}$ satisfies constrained equal-budget choice equivalence and Maskin monotonicity if and only if $\bar{f}_{\alpha}=C W_{E Q} \cdot{ }^{21}$ Observe that $W E_{E Q} \subseteq C W_{E Q}$. In addition, if preferences are strongly monotonic, then for any $(R, \bar{\omega}) \in E^{N}$ and any $x \in C W_{E Q}(R, \bar{\omega})$, allocation $x$ is Pareto efficient. An important observation is that if $\alpha \neq \frac{\bar{\omega}}{n}$, then $\bar{f}_{\alpha}$ is

\footnotetext{
${ }^{20}$ Thomson (1999) in fact shows that $C W_{E Q}$ is the minimal Maskin monotonic extension of $W_{E Q}$.

${ }^{21}$ Observe that a selection of the Constrained equal-income Walrasian rule is not necessarily Maskin monotonic.
} 
neither a subcorrespondence nor a supercorrespondence of its unconstrained counterpart $f_{\alpha}$.

Remark 3: An interesting extension would be to consider fair solutions suggested in the literature and to construct the family of choice sets generating them as equal-opportunity equivalent solutions. If they satisfy implementation via some game theoretic solution concept, then the components of the family of choice sets (e.g. bundles, allocations etc...) can be used as part of the strategy sets of agents. For instance, suppose that a given solution is Nash implementable using a family of choice sets $\mathcal{B}$. Then, we conjecture that the announcements of preference profiles in canonical mechanisms such as Abreu and Sen (1990) for subgame perfect implementation; Maskin (1999) or Bochet (2005b) for Nash implementation; can be replaced by announcements of choice sets. This work would suggest a close connection between equal-opportunity equivalence and the possibility to perform strategy space reductions from infinite to finite spaces in canonical mechanisms used to $\mathcal{S C}$-implement an allocation rule.

\section{References:}

1. Abreu D., Sen A., 1990. "Subgame Perfect Implementation: a Necessary and Almost Sufficient Condition.". Journal of Economic Theory, 50, 285-299.

2. Bochet O., 2005a. "Implementation of the Walrasian Correspondence: The Boundary Problem.". Mimeo, Maastricht University.

3. Bochet O., 2005b. "Nash Implementation with Lottery Mechanisms." Forthcoming in Social Choice and Welfare.

3. Bochet O., Maniquet F., 2005. "Virtual Nash Implementation with Admissible Supports.". Mimeo, Maastricht University.

4. Dutta B., Sen A. and Vohra R., 1995. "Nash Implementation through Elementary Mechanisms in Economic Environments.", Review of Economic Design, 1, 173-204.

5. Foley D., 1967. "Resource Allocation and the Public Sector." Yale Economic Essays, 7, 45-98.

6. Gevers L., 1986. "Walrasian Social Choice: Some Simple Axiomatic Approaches." in Social Choice and Public Decision Making. Heller W. et al. (eds.) Cambridge University Press: cambridge (vol 1), 97-114.

7. Jackson J., 1992. "Implementation in Undominated Strategies: A Look at Bounded Mechanisms." Review of Economic Studies, 59, 757-775. 
8. Maskin E., 1999. "Nash Equilibrium and Welfare Optimality." Review of Economic Studies, 66, 23-38.

9. Maniquet F., 2003. "Implementation of Allocation Rules under Perfect Information" Social Choice and Welfare, 21, 323-346.

10. Moore J. and Repullo R., 1988. "Subgame Perfect Implementation." Econometrica, 56, 1191-1120.

11. Nicolo A. and Perea A., 2005. "Monotonicity and Equal-Opportunity Equivalence in Bargaining." Mathematical Social Sciences, 49, 221-243.

12. Pazner E., Schmeidler D., 1978. "Egalitarian-Equivalent Allocations: A New Concept of Economic Equity." Quarterly Journal Of Economics, 92, 671-687.

13. Thomson W., 1988. "A Study of Choice Correspondences in Economies with a Variable Number of Agents." Journal of Economic Theory, 46, 237254 .

14. Thomson W., 1994. "Notions of Equal, or Equivalent, Opportunities." Social Choice and Welfare, 11, 137-156.

15. Thomson W., 1999. "Monotonic Extensions on Economic Domains." Review of Economic Design, 4, 13-33.

16. Thomson W., 2005. "Divide and Permute and the Implementation of Solutions to the Problem of Fair Division." Games and Economic Behavior, $52,186-200$. 